\section{Dual-reporter assay using two secreted luciferase genes}

\author{
Chun Wu ${ }^{1}$, Chie Suzuki-Ogoh ${ }^{1}$, and Yoshihiro Ohmiya ${ }^{1,2}$ \\ ${ }^{1}$ National Institute for Advanced Industrial Science and Technology (AIST), Osaka and \\ ${ }^{2}$ Hokkaido University, Sapporo, Japan
}

BioTechniques 42:290-292 (March 2007)

doi 10.2144/000112428

Luciferase is used as a reporter enzyme to estimate gene expression in prokaryotic or eukaryotic cells because the amount of luciferase present is correlated with light intensity in the presence of excess luciferin (1). Furthermore, its sensitivity and range of linear responses are superior to those of other typical reporters (2). When the luciferase gene containing a target promoter region is transfected into target cells using a plasmid, expression of luciferase can be estimated from bioluminescence after 1 or 2 days. For practical use, two or three promoters are used for the target and control genes because the expression of a reporter enzyme depends on conditions in the cell, which can be estimated from expression of a control gene. The dual luciferase reporter assay system (www.promega.com/tbs/tm040/tw040. $\mathrm{html}$ ) involves the use of firefly luciferase for monitoring gene expression and another type of luciferase [e.g., sea pansy (Renilla) luciferase] as an internal control. Recently, Nakajima et al. developed a tricolor-reporter in vitro assay system in which expression of three genes is monitored simultaneously by splitting emissions from green-, orange-, and red-emitting beetle luciferases using optical filters (3). However, neither in vitro assay system is suitable for high-throughput analysis of multiple gene expression, because they involve sample lysis or require special equipment, such as a filtered luminometer. In this report, we describe a new dual-reporter assay involving two secreted luciferases that does not require lysis or special equipment.

The marine ostracod crustacean Cypridina and the marine copepod Gaussia secrete light-blue luminescent luciferases into seawater $(4,5)$. When cDNA for Cypridina luciferase (CLuc; $\lambda \max =460 \mathrm{~nm})$ is transfected into mammalian cells, the CLuc is secreted into the medium via the endoplasmic reticulum (ER) and the Golgi complex (6). Gaussia luciferase (GLuc; $\lambda \max =$
A

B
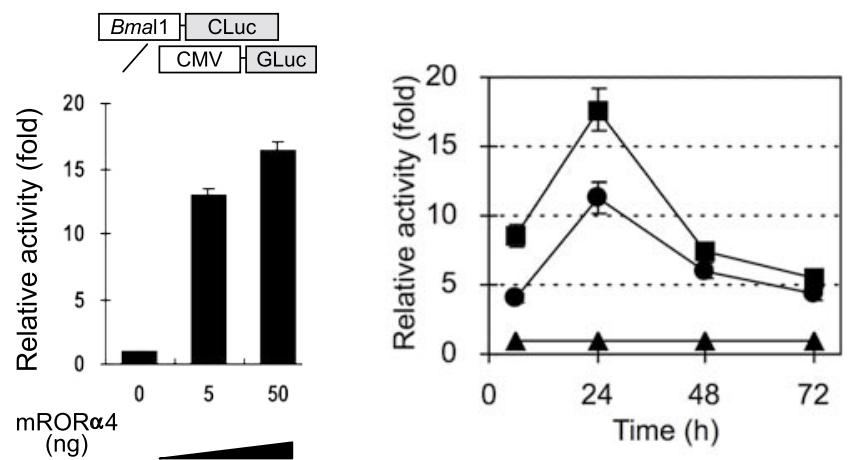

C

D
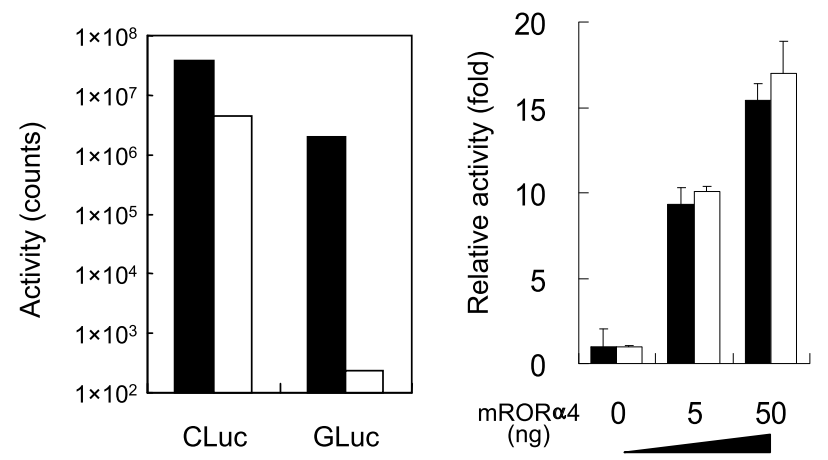

Figure 1. A dual secreted reporter system. (A) Monitoring of mROR $\alpha 4$ dosedependent induction of Bmal1 promoter transcription after incubation for $24 \mathrm{~h}$. Plasmid DNAs (50 ng pBmal1-CLuc and 0, 5, and $50 \mathrm{ng}$ pCR3.1-mROR $\alpha 4$ as transactivator; $5 \mathrm{ng}$ pCMV-GLuc with constitutive expression of GLuc for normalizing transfection efficiency) and $105 \mathrm{ng}$ pBluescript ${ }^{\circledR}$ SK (-) phagemid (Stratagene, La Jolla, CA, USA) as an additive were transfected into NIH 3T3 cells in 24-well plates. After $24 \mathrm{~h}$, two partial aliquots of medium were dispensed into two tubes. For assay of CLuc activity, an aliquot was reacted with Cypridina luciferin solution (1 $\mu \mathrm{M}$ Cypridina luciferin, $60 \mathrm{mM}$ phosphate buffer, $\mathrm{pH}$ 6.4, $0.3 \mathrm{M}$ sodium ascorbate, and $20 \mathrm{mM} \mathrm{Na}_{2} \mathrm{SO}_{3}$ ). For assay of GLuc activity, the other aliquot was reacted with coelenterazine solution $(1 \mu \mathrm{M}$ coelenterazine, $0.1 \mathrm{M}$ Tris- $\mathrm{HCl}$ buffer, $\mathrm{pH} 7.4$, and $0.3 \mathrm{M}$ sodium ascorbate). (B) Time course of mROR $\alpha 4$ dose-dependent induction of Bmal1 promoter transcription. Transfected cells were added by 0 (closed triangle), 5 (closed circle), and $50 \mathrm{ng}$ (closed square) pCR3.1-mROR $\alpha 4$ as transactivator. After 6, 24, 48, and $72 \mathrm{~h}$, two partial aliquots of medium were dispensed into two tubes. For assay of CLuc or GLuc activities, $50 \mu \mathrm{L}$ sample were reacted with Cypridina luciferin or coelenterazine solutions. (C) Effect of sodium dodecyl sulfate (SDS) on the activities of CLuc and GLuc. For assay of CLuc activity, $50 \mu \mathrm{L}$ CLuc sample were reacted with $50 \mu \mathrm{L}$ Cypridina luciferin solution (black column) and with $10 \mu \mathrm{L} \mathrm{1 \%}$ SDS (white column). For assay of GLuc activity, $50 \mu \mathrm{L}$ GLuc sample were reacted with $50 \mu \mathrm{L}$ coelenterazine solution (black column) and with $10 \mu \mathrm{L} 1 \%$ SDS (white column). (D) Monitoring of mROR $\alpha 4$ dosedependent induction of Bmal1 promoter transcription in one- and two-tube procedures. Plasmid DNAs (50 ng pBmal1-CLuc and 0, 5, and $50 \mathrm{ng}$ pCR3.1mROR $\alpha 4$ as transactivator; 5 ng pCMV-GLuc with constitutive expression of GLuc for normalizing transfection efficiency) and $105 \mathrm{ng}$ pBluescript SK (-) phagemid as an additive were transfected into NIH 3T3 cells in 24-well plates. For the two-tube dual-reporter assay procedure, two partial aliquots of medium were dispensed into two tubes incubated after $24 \mathrm{~h}$ (black column). For assay of CLuc activity, an aliquot was reacted with Cypridina luciferin solution. For assay of GLuc activity, the other aliquot was reacted with coelenterazine solution. For the one-tube dual-reporter assay procedure, an aliquot of medium was dispensed into one tube incubated after $24 \mathrm{~h}$ (white column). For assay of GLuc activity, the same aliquot was reacted with coelenterazine solution. At the next step, the bioluminescence of GLuc-coelenterazine reaction was stopped by adding $1 \%$ SDS (final concentration $0.1 \%$ ). Finally, for assay of CLuc activity, an aliquot was reacted with Cypridina luciferin solution. GLuc, Gaussia luciferase; CLuc, Cypridina luciferase; CMV, cytomegalovirus. 


\section{Benchmarks}

$480 \mathrm{~nm}$ ) is secreted into the medium via a similar mechanism when its cDNA is transfected into mammalian cells. The activities of both luciferases in the culture medium can be easily quantified from light intensity after reaction with their substrates [CLuc reacts with Cypridina luciferin (7), and GLuc reacts with coelenterazine]. The bioluminescence systems did not crossreact, and the half-times for decay were similar ( $>60 \mathrm{~h}$ at room temperature; data not shown). Thus, the activities of the two secreted luciferases can be measured separately and accurately.

The plasmid vectors pBmal1-CLuc, which contain a Bmal1 promoter as a target gene, and pCMV-GLuc (New England Biolabs, Ipswich, MA, USA), which was used as a control gene, were transfected into NIH 3T3 cells. Figure 1A shows the dose-dependent induction of Bmal1 promoter-driven transcription compared with induction of the cytomegalovirus (CMV) promoter by ROR $\alpha 4$ during a 24-h incubation. CLuc activity increased in response to ROR $\alpha 4$, which is a transactivator of the Bmal1 gene (8). Furthermore, as shown in Figure 1B, the time course of Bmal1 promoter activity is dose-dependent on ROR $\alpha 4$. The dose dependency was similar at each point. Above a 48-h incubation, the accumulation or induction effect of ROR $\alpha 4$ is less than those in the early phase, although the bioluminescence activities increase gradually. This phenomenon may be reflected by the characteristic of ROR $\alpha 4$ or cell conditions.

In comparison with previous reporter assay systems, our new dual-reporter assay has several advantages: (i) it can be carried out sequentially over periods of hours to days using the same cell population; (ii) activities of two luciferases are relatively high and can be measured in small volume $(<10$ $\mu \mathrm{L}$ medium); and (iii) the procedure is simple and does not require special equipment. Our dual assay is thus suitable for high-throughput analysis of gene transcription in living cells.

However, this pro-cedure involves the use of two tubes for one assay, which requires accurate separation of the sample. As sodium dodecyl sulfate (SDS; final concentration $0.1 \%$ ) inhibited GLuc activity $(<0.01 \%)$ but not CLuc activity (Figure 1C), we developed a one-tube dual-reporter assay. The procedure was as follows: (i) GLuc activity was measured with coelenterazine; (ii) the bioluminescence of GLuc-coelenterazine reaction was stopped by adding 10\% SDS (final concentration $0.1 \%$ ); and (iii) CLuc activity was measured using Cypridina luciferin. Results obtained using the one-tube procedure were similar to those obtained using the two-tube procedure (Figure 1D).

This reporter assay system could be used for detailed analysis of the transcriptome and the promoterome, as well as for screening of new drugs or detection of harmful chemicals.

\section{ACKNOWLEDGMENTS}

We thank M. Ikeda for kindly providing us with the plasmid vector; $Y$. Nakajima and K. Yamagishi for helpful discussion and suggestions.

\section{COMPETING INTERESTS STATEMENT}

The authors declare no competing interests.

\section{REFERENCES}

1. Ohmiya, Y. 2005. Basic and applied aspects of color tuning of bioluminescence systems. Jpn. J. Appl. Phys. 22:1543-1545.

2. Naylor, L.-H. 1999. Reporter gene technology: the future looks bright. Biochem. Pharmacol. 58:749-757.

3. Nakajima, Y., T. Kimura, K. Sugata, T. Enomoto, T. Asakawa, H. Kubota, M. Ikeda, and Y. Ohmiya. 2005. A multicolor luciferase assay system, one-step monitoring of multiple gene expressions with a single substrate. BioTechniques 38:891-894.

4. Nakajima, Y., K. Kobayashi, K. Yamagishi, T. Enomoto, and Y. Ohmiya. 2004. cDNA cloning and characterization of a secreted luciferase from the luminous Japan ostracod, Cypridina noctiluca. Biosci. Biotechnol. Biochem. 68:565-570.

5. Verhaegent, M. and T.K. Christopoulos. 2002. Recombinant Gaussia luciferase. Overexpression, purification, and analytical application of a bioluminescent reporter for DNA hybridization. Anal. Chem. 74:43784385.

6.Inouye, S., Y. Ohmiya, Y. Toya, and F.I. Tsuji. 1992. Imaging of luciferase secretion from transformed Chinese hamster ovary cells. Proc. Natl. Acad. Sci. USA 89:9584-9587.

7.Wu, C. K. Kawasaki, S. Ohgiya, and Y. Ohmiya. 2006. Syntheses and evaluation of the bioluminescent activity of (S)-Cypridina luciferin and its analogs, Tetrahedron Lett. 47:753-756

8. Yamagishi, K., T. Enomoto, and Y. Ohmiya. 2006. Perfusion-culture-based secreted bioluminescence reporter assay in living cells. Anal. Biochem. 354:15-21.
Received 29 November 2006; accepted 17 January 2007.

Address correspondence to Yoshihiro Ohmiya, Research Institute for Cell Engineering, National Institute of Advanced Industrial Science and Technology (AIST), Osaka, 563-8577, Japan. e-mail: y-ohmiya@aist.go.jp

To purchase reprints of this article, contact: Reprints@BioTechniques.com 